\title{
Real-time 2-D Phased Array Vector Flow Imaging
}

Holbek, Simon; Hansen, Kristoffer Lindskov; Fogh, Nikolaj; Moshavegh, Ramin; Olesen, Jacob Bjerring; Bachmann Nielsen, Michael; Jensen, Jørgen Arendt

Published in:

IEEE Transactions on Ultrasonics, Ferroelectrics and Frequency Control

Link to article, DOI:

10.1109/TUFFC.2018.2838518

Publication date:

2018

Document Version

Peer reviewed version

Link back to DTU Orbit

Citation (APA):

Holbek, S., Hansen, K. L., Fogh, N., Moshavegh, R., Olesen, J. B., Bachmann Nielsen, M., \& Jensen, J. A. (2018). Real-time 2-D Phased Array Vector Flow Imaging. IEEE Transactions on Ultrasonics, Ferroelectrics and Frequency Control, 65(7), 1205-1213. https://doi.org/10.1109/TUFFC.2018.2838518

\section{General rights}

Copyright and moral rights for the publications made accessible in the public portal are retained by the authors and/or other copyright owners and it is a condition of accessing publications that users recognise and abide by the legal requirements associated with these rights.

- Users may download and print one copy of any publication from the public portal for the purpose of private study or research.

- You may not further distribute the material or use it for any profit-making activity or commercial gain

- You may freely distribute the URL identifying the publication in the public portal 


\title{
Real-time 2-D Phased Array Vector Flow Imaging
}

\author{
Simon Holbek ${ }^{1,2}$, Kristoffer Lindskov Hansen ${ }^{3,4}$, Nikolaj Fogh ${ }^{1}$, Ramin Moshavegh ${ }^{1,2}$, Jacob Bjerring Olesen ${ }^{1,2}$, \\ Michael Bachmann Nielsen ${ }^{3,4}$, and Jørgen Arendt Jensen ${ }^{2}$ \\ ${ }^{1}$ BK Ultrasound, Mileparken 34, 2730 Herlev, Denmark \\ ${ }^{2}$ Center for Fast Ultrasound Imaging, Dept. of Elec. Eng., Bldg. 349, Technical University of Denmark, \\ 2800 Kgs. Lyngby, Denmark \\ ${ }^{3}$ Department of Diagnostic Radiology, Copenhagen University Hospital, DK 2100 Copenhagen, Denmark \\ ${ }^{4}$ Department of Clinical Medicine, University of Copenhagen, DK 2100 Copenhagen, Denmark
}

\begin{abstract}
Echocardiography examination of the blood flow is currently either restricted to $1-D$ techniques in real-time or experimental off-line 2-D methods. This paper presents an implementation of transverse oscillation for real-time 2-D vector flow imaging (VFI) on a commercial BK Ultrasound scanner. A large field-of-view (FOV) sequence for studying flow dynamics at 11 frames per second (fps) and a sequence for studying peak systolic velocities (PSV) with a narrow FOV at 36 fps were validated. The VFI sequences were validated in a flow-rig with continuous laminar parabolic flow and in a pulsating flow pump system before being tested in vivo, where measurements were obtained on two healthy volunteers. Mean PSV from 11 cycles was $155 \mathrm{~cm} \mathrm{~s}^{-1}$ with a precision of $\pm 9.0 \%$ for the pulsating flow pump. In vivo, PSV estimated in the ascending aorta was $135 \mathrm{~cm} \mathrm{~s}^{-1} \pm 16.9 \%$ for 8 cardiac cycles. Furthermore, in vivo flow dynamics of the left ventricle and in the ascending aorta were visualized. In conclusion, angle independent 2-D VFI on a phased array has been implemented in real-time, and it is capable of providing quantitative and qualitative flow evaluations of both complex and fully transverse flow.
\end{abstract}

\section{INTRODUCTION}

Assessment of cardiac function is often evaluated with echocardiography [1]. Small phased array transducers are the preferred choice for echocardiography, since the footprint should fit between the ribs of the patient, and a large field-ofview (FOV) is required to image the dynamics of entire heart chambers. Standard echocardiography examinations provide clinicians with a real-time cross-sectional image of the heart with the option of overlaying the image with 1-D blood flow information using color Doppler techniques or by using 1-D spectral Doppler methods, which provide velocity estimates in a range gate specified by the user. Access to information about flow dynamics in the heart chambers and to cardiac output estimates have become important for assessment of the physiological functioning of the heart and for cardiac surveillance [2]-[5].

However, one of the limitations with the current 1-D velocity estimators is their sensitivity to false estimates [6], [7], which commonly arise from the manual angle correction by the operator, also denoted the angle dependency problem. Furthermore, conventional 1-D spectral Doppler assumes a fixed beam-to-flow angle throughout the entire cardiac cycle, which is not always the case [8]. The fixed-angle assumption also hinders quantitative examination of blood flow velocities in the heart chambers, where rapidly changing flow dynamics are present [9].

The angle dependency problem can be avoided using 2D vector flow imaging (VFI) [10]-[14]. With VFI, the sonification angle is in principle irrelevant, although the actual performance might be reflected in the beam-to-flow angle.

Recently, several approaches have been reported in the literature for phased array VFI. A well known VFI technique that has been applied on a phased array transducer is speckle tracking [15], which has been implemented for both 2-D [16], [17] and 3-D imaging [18] in echocardiography. Also directional beamforming methods have successfully been applied to estimate both 2-D [19] and 3-D VFI [20] on 1-D and 2-D phased array transducers, respectively. Additionally, Doppler vortography has been proposed to visualize vortices forming in the left ventricle [21]-[23]. An overview of all major methods for vector flow imaging for sequential data acquisitions is found in [24] and for parallel acquisition in [25].

This paper uses the transverse oscillation (TO) method [26], [27] for estimating 2-D VFI on a phased array transducer. Experimental 2-D VFI results for cardiac applications have previously been reported in the literature for a 1-D phased array transducer [28] and in a 3-D expansion of the technique for blood flow estimation in superficial vessels using a 2D phased array matrix probe [29], [30]. Moreover, the TO method has also been used for tissue displacement estimation [31], which was further developed on a phased array for 2-D motion estimation in echocardiography [32]. However, until today, none of the developed methods for phased array VFI have been implemented on a scanner for real-time rendering. Mindray have implemented VFI using the approach by Yu et al [33] based on multi-angle velocity estimation, and GE has introduced blood speckle imaging based on plane wave transmissions and speckle tracking. Both implementations are, however, not real time, and they have an acquisition phase and a display stage due to their high calculation demands.

In this paper, we present a setup for real-time phased array VFI using the TO method, which currently is implemented on a linear and a curved array. The approach is validated in two phantom setups (constant parabolic flow and pulsating flow) and in vivo on two healthy volunteers.

\section{1}




\section{Materials \& Methods}

The following section describes the applied materials and reviews the methods used in this study. An overview of the specifications is found in Table I.

\section{A. Scanner setup}

A commercially available ultrasound scanner (BK 5000, BK ultrasound, Herlev, Denmark) was used along with a phased array transducer with a frequency range from $1-5 \mathrm{MHz}(5 \mathrm{P} 1$, BK Ultrasound, Herlev, Denmark) for transthoracic cardiac application.

\section{B. Transverse Oscillation velocity estimator}

The transverse oscillation method was used as the velocity estimator in this work and is based on the idea introduced by Jensen and Munk [26]. This paper only reviews the basic principles of the phase shift estimator, but an in depth introduction can be found in the literature [10].

Similar to the conventional axial auto-correlation velocity estimator [34], the TO estimator relies on generating a lateral oscillating field, from which the lateral velocity component can be estimated. The lateral oscillating field is generated in receive by applying two rectangular apodization functions separated by the distance $d_{x}$ in receive. The depth dependent lateral wavelength $\lambda_{x}(z)$ is then given by

$$
\lambda_{x}(z)=2 \lambda_{z} \frac{z}{d_{x}}
$$

where $z$ is the depth and $\lambda_{z}$ the axial wavelength. The theoretical derived lateral wavelength has previously shown to be biased, and hence, an optimization procedure has to be performed to find the unbiased wavelength [35]. The apodization profiles used in this study were two separated 16 element rect-functions.

Steered focused emissions were used in this study, and for each transmit event three lines were beamformed. One line used for the axial estimator was beamformed along $(0, z)$, and two lines used for the lateral velocity estimator were beamformed along $\left( \pm \lambda_{x}(z) / 8,0, z\right)$. The maximum detectable lateral velocity without reaching the aliasing limit is then given by

$$
v_{x(z)_{\max }}= \pm \frac{\lambda_{x}(z) f_{p r f}}{4 k}
$$

where $f_{p r f}$ is the effective system pulse repetition frequency (PRF) and $k$ the applied lag in the auto-correlation function.

\begin{tabular}{lc} 
Parameter & Value \\
\hline Scanner & BK 5000 \\
Transducer & Phased array \\
Number of elements & 96 \\
Width of TO peaks & 16 elements \\
Tx center frequency & $2.7 \mathrm{MHz}$ \\
Pulse periods & 3 \\
F-number & 4.2 \\
Lateral oscillation wavelength & $5.8 \mathrm{~mm}$ \\
Pulse repetition frequency & $3.1 \mathrm{kHz}$ \\
Moving average & 4 frames
\end{tabular}

Table I: Major parameters for the vector flow imaging.
With the current setup, this implies that at $8 \mathrm{~cm}$ depth with $\lambda_{x}=5.8 \mathrm{~mm}, k=1$ and at an $f_{p r f} 3.1 \mathrm{kH}$, a theoretical lateral velocity of $\pm 4.5 \mathrm{~m} \mathrm{~s}^{-1}$ can be detected.

\section{Beamforming}

Following the lateral wavelength optimization routine [35], a $\lambda_{x}$ was determined at the focal depth of $8 \mathrm{~cm}$. Based on the trigonometric relation between the depth of investigation and $\lambda_{x}$, a beamforming angle $\pm \theta$ was estimated. The beamforming angle was fixed for all VFI transmit events. An illustration of the setup is seen in Fig. 1.

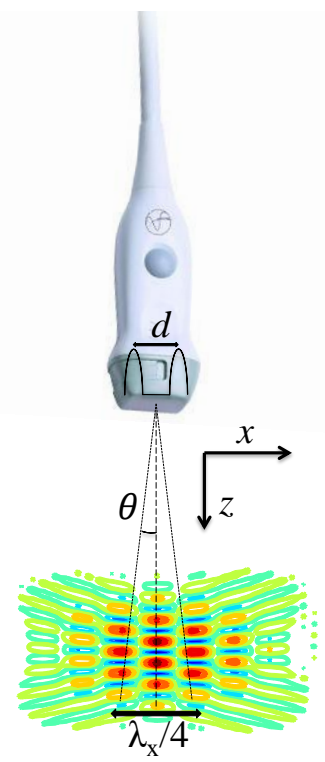

Figure 1: Illustration of the TO beamforming setup. Two lines are beamformed at a fixed angle $\pm \theta$ relative to the focal point for estimation of the lateral velocity component. Here, a receive apodization consisting of two peaks separated by a distance $d$ is used. A third line is beamformed along the direction of the focal point for estimation of the axial velocity component.

\section{VFI sequences}

A 3-cycle pulse transmitted at $2.7 \mathrm{MHz}$ with an F-number of 4.2 was used for the flow emissions. Two different interleaved line-by-line VFI sequences were used in this study as illustrated in Fig 2;

1) VFI sequence with 68 transmit lines providing a large Field-of-view (FOV) at 11 frames per second (fps) to study qualitative heart chamber flow complexity.

2) VFI sequence with 8 transmit lines providing a small FOV and a higher frame rate of $36 \mathrm{fps}$ to study quantitative peak velocities.

The depth of the B-mode image was $15 \mathrm{~cm}$ and for flow estimates $10.6 \mathrm{~cm}$ in both setups. 12 emissions were used for flow estimate/frame, and a moving average of size 4 frames was applied continuously on the estimates before the final rendering. 

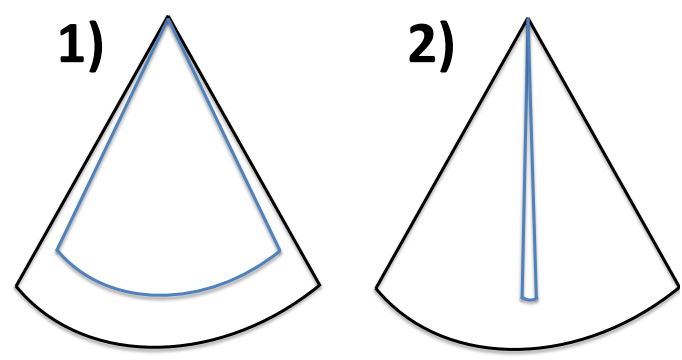

Figure 2: The two VFI sequences used in the study. VFI data are present within the area encapsulated by the blue lines and B-mode information is present inside the larger black area. 1) provides a large FOV at a low frame rate. 2) Provides a small FOV at a high frame rate.

\section{E. Echo cancellation and filtering}

For stationary echo cancellation a simple removal of the DC component was achieved by subtracting the mean of the eight transmissions from the ensemble window. The resulting flow estimates were later filtered spatially through a two-fold process. Initially, an anti-aliasing algorithm was imposed to minimize the risk of any inexpedient discontinuities in the estimated flow field before applying the smoothing algorithm. The smoothing algorithm consisted of a 3-point median filter in the axial direction, and a $5 \times 5$-point median filter for the lateral direction.

\section{F. Data acquisition}

Data acquisition of the real-time VFI displayed on the ultrasound scanner was performed, when the operator pushed a dedicated button in the ultrasound scanner user interface for data streaming. A continuous stream of processed B-mode and VFI frames were stored on the scanners' hard drive until the operator ended the data dump via the interface. The data were subsequently transferred to a Linux PC, where off-line visualization and data analysis were performed with an inhouse built visualization tool.

\section{G. Visualization tool}

Although real-time rendering of the 2-D velocity estimates was present on the scanner, an in-house developed MATLABbased visualization GUI was used to analyze all the stored cineloop data off-line. Data were loaded in the GUI and visualized as seen in Fig. 3. Data were processed using either of two developed methods for analysis: A single point analyzer, which estimates the flow in a user-specified position through time. This was only used for analyzing pulsating flow, i.e during experimental flow pump measurements and in in vivo measurements. At the user-specified location, the velocities through time were found, and an automated algorithm was applied to identify multiple heart cycles and align the estimates, such that the mean cardiac cycle \pm one standard deviation (STD) could be inspected. The second method was a multi line approach, which calculated the mean flow profile along user-defined start and end steering directions as illustrated in Fig. 3.

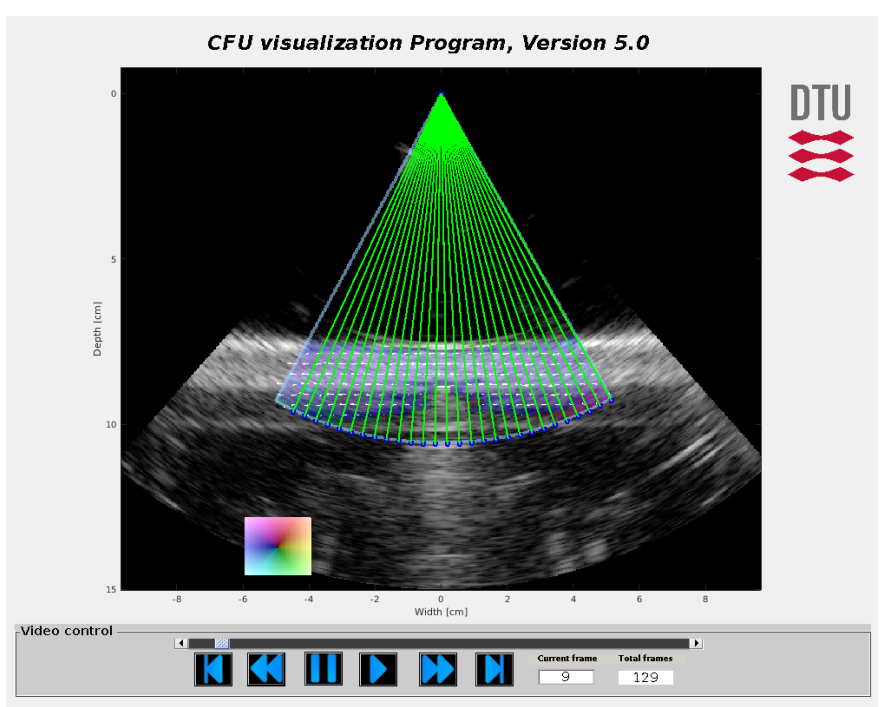

Figure 3: Screen shot from the visualization tool where velocity and angle analysis can be made for multiple steering angles illustrated by the green lines. The flow direction and the magnitude are given by the length and direction of the white arrows and the color overlay.

\section{MeAsurement SETUP}

In all phantom measurements, the transducer was placed in a fixture initially at $90^{\circ}$ relative to the straight vessel, which could be angulated in steps of $5^{\circ}$. Furthermore, the fixture could be adjusted to vary the distance from the transducer surface to the measuring site.

\section{A. Flow-Rig}

Measurements on steady laminar flow were performed in an in-house built flow-rig system. An $1.2 \mathrm{~m}$ long inlet ensured that a parabolic flow profile was present at the measuring site. Blood mimicking fluid was driven in the system by a MAG 1100 flow meter (Danfoss, Hesselager, Denmark), which provided a volumetric flow rate of $Q$. At the measuring site, the rubber vessel $(\varnothing=12 \mathrm{~mm})$ was immersed into a water tank containing demineralized water. The flow rate was set to $102.6 \mathrm{~mL} \mathrm{~min}^{-1}$ translating to a peak velocity of $50 \mathrm{~cm} \mathrm{~s}^{-1}$, and the center of the vessel was located at $8 \mathrm{~cm}$ depth. The large FOV sequence (sequence 1) was used in the flow-rig measurements.

\section{B. Pulsatile Flow Pump}

A flow system (CompuFlow 1000, Shelley Medical Imaging Technologies, Toronto, Canada) was used to generate a predefined time-varying carotid flow waveform with user defined output flow rate. The cycle time was $0.84 \mathrm{~s}$ translating to 71 beats per minute. The manufacturer specified flow rate accuracy of the system was $\pm 3 \%$. The flow pump was connected to a customized tissue mimicking phantom (Dansk Fantom Service, Frederikssund, Denmark), containing a straight-vessel $(\varnothing=4 \mathrm{~mm})$ with the center located $6.6 \mathrm{~cm}$ beneath the transducer's surface. Measurements were conducted with a 


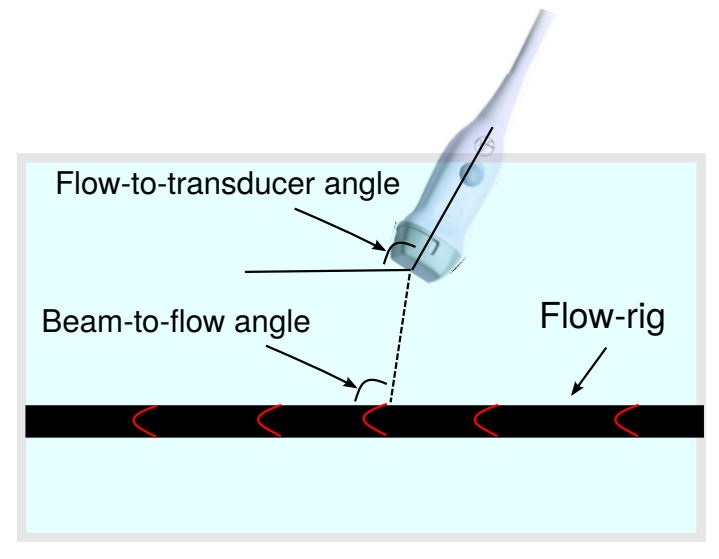

Figure 4: Illustration of the flow-rig measurement setup containing a parabolic flow. The flow-to-transducer angle is denoted by the solid black line and the beam-to-flow angle is denoted by the steered dotted black line. mean output flow rate of $5.13 \mathrm{~mL} /$ cycle. The small FOV sequence (sequence 2) was used in the pulsatile flow pump measurements.

\section{FDA Limits}

Intensity measurements were performed for the two sequences: 1) $I_{\text {spta }}=1121 \mathrm{~mW} / \mathrm{cm}^{2}$ and $\left.\mathrm{MI}=1.42 .2\right) I_{\text {spta }}=$ $5565 \mathrm{~mW} / \mathrm{cm}^{2}$ and $\mathrm{MI}=0.95$. All within current FDA limits [36]. Furthermore, the measured rise in surface temperature also met the FDA limits for both sequences.

\section{In vivo Measurements}

The in vivo study has been approved by the local ethics committee (no. 17020259). In vivo measurements were performed on a healthy 31 year old male (volunteer 1), and on a healthy 43 year old male (volunteer 2 ). The volunteers had been resting for 10-15 min in supine position prior to the examination. A parasternal long axis (PLAX) view of the ascending aorta was obtained both with the large FOV and the small FOV sequence on volunteer 1, and a large FOV measurement was obtained of the left ventricle on volunteer 2 . The measurements were in working with ultrasonic VFI.

\section{RESUlTS}

\section{A. Flow-Rig Measurements}

Data from the flow-rig measurement were loaded into the visualization GUI. Mean absolute velocity profiles with bias, flow angles, and STD were calculated based on the first 100 frames of acquisition. Profiles were calculated from the steering angles $-25^{\circ}$ to $25^{\circ}$ in steps of $1^{\circ}$ for the flow-totransducer angles $90^{\circ}$ and $115^{\circ}$, where the flow-to-transducer angle is the relative angle between the flow in the straight vessel and the transducer surface. A schematic illustration of the setup is seen in Fig.4.

All metrics were calculated at the position inside the vessel, where the highest mean absolute velocity was found (Fig. 5). performed by a radiologist (KLH) with 10 years of experience

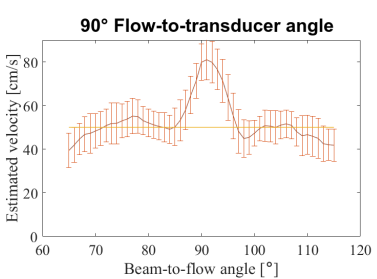

(a)

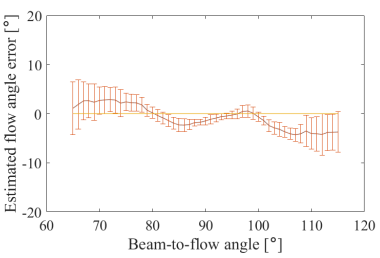

(c)

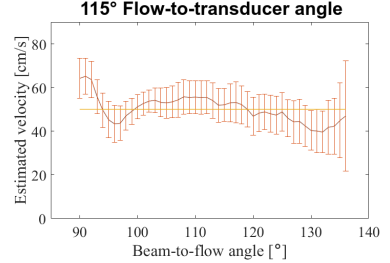

(b)

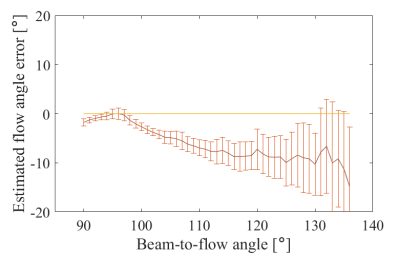

(d)
Figure 5: Results from flow-rig measurements using a large FOV sequence. All results are shown as a function of relative beam-to-flow angle. a) Estimated mean velocity for a $90^{\circ}$ flow-to-transducer angle. c) Estimated flow angle error for a $90^{\circ}$ flow-to-transducer angle. b) Estimated mean velocity for a $115^{\circ}$ flow-to-transducer angle. d) Estimated flow angle error for a $115^{\circ}$ flow-to-transducer angle. All graphs are shown with \pm one STD (error bars) and the expected value (orange line).

The highest bias is seen at a relative beam-to-flow angle close to $90^{\circ}$, which reaches $62 \%$ and $29 \%$ at the respective flowto-transducer angles of $90^{\circ}$ (Fig. 5a) and $115^{\circ}$ (Fig. 5b). The sharp rise in bias around a $90^{\circ}$ relative beam-to-flow angle is further discussed in Section V. As the flow direction gradually deviates from a purely transverse flow, the STD on both the velocity estimate and the estimated flow angle increases. For a $115^{\circ}$ flow-to-transducer angle (Fig. 5b), the STD on the velocity is lowest at a $102^{\circ}$ beam-to-flow angle $( \pm 9.3 \%)$ and highest at $136^{\circ}$ ( $\pm 53.8 \%$ ). Similarly, the STD on the flow angle estimate rises from $\pm 0.5^{\circ}$ to $\pm 12.2^{\circ}$ at the respective beam-to-flow angles of $92^{\circ}$ and $136^{\circ}$ (Fig. 5d).

Results for selected velocity and flow angle profiles at $90^{\circ}$ and $115^{\circ}$ flow-to-transducer angles at $-25^{\circ}$ and $0^{\circ}$ steering angles are seen in Figs. 6 and 7. Here, a reverberation artefact is seen as a second spike in the estimated velocity magnitude, located after the true peak of the parabolic flow. The artefact is seen in Fig. $6 \mathrm{a}$ and $7 \mathrm{~b}$ around $9 \mathrm{~cm}$ depth. The artefact did not influence the estimated theoretical parabolic peak velocities.

\section{B. Flow Pump Measurements}

A total of $10 \mathrm{~s}$ of data were recorded with the small FOV sequence corresponding to 11 cycles. The results for the estimated velocity magnitude in the center of the vessel are shown in Fig. 8a. Coherent alignment of the identified 11 cycles gave a mean peak velocity of $155 \mathrm{~cm} \mathrm{~s}^{-1} \pm 9.0 \%$ (Fig. $8 \mathrm{~b}$. The estimated flow angle through time is seen in Fig. 8c and the mean angle throughout the entire cycle was $90.4^{\circ}$ with an STD of $\pm 0.4^{\circ}$ (Fig. 8d). 


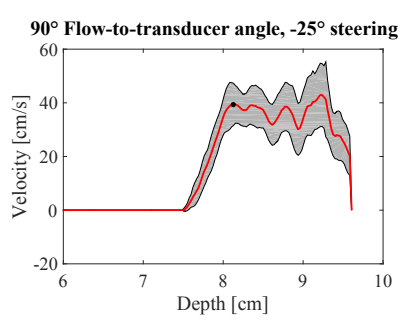

(a)

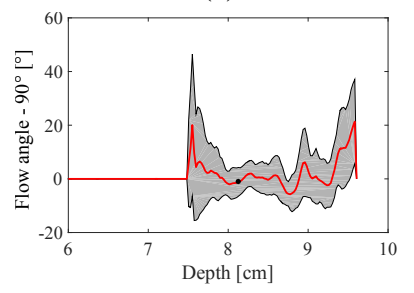

(c)

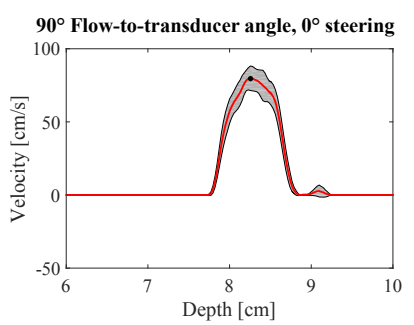

(b)

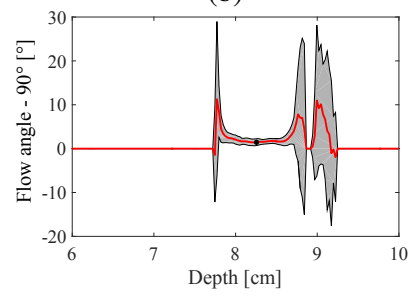

(d)
Figure 6: Estimated flow rig mean velocities and mean angles (red curve) \pm one STD (grey area) based on 100 frames for a $90^{\circ}$ beam-to-flow angle for a $-25^{\circ}$ steering angle (a),(c), and for a $0^{\circ}$ steering angle (b), (d).

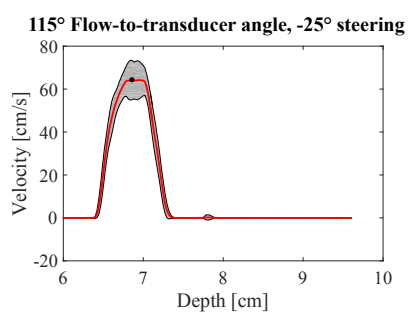

(a)

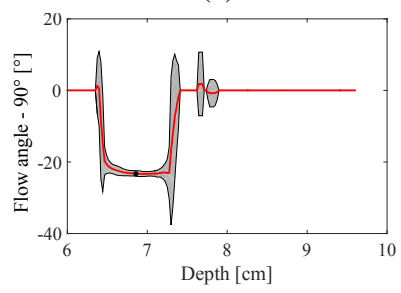

(c)

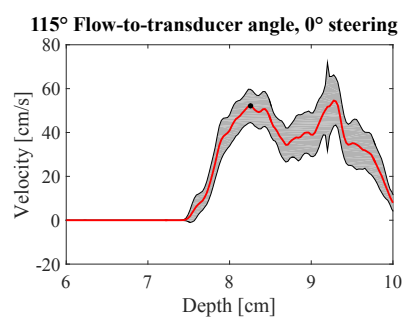

(b)

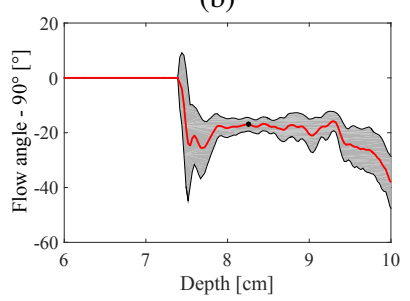

(d)
Figure 7: Estimated flow rig mean velocities and mean angles (red curve) \pm one STD (grey area) based on 100 frames for a $115^{\circ}$ flow-to-transducer angle for a $-25^{\circ}$ steering angle (a),(c), and for a $0^{\circ}$ steering angle (b),(d).

\section{In Vivo}

Results from the PLAX view of the ascending aorta measured on volunteer 1 are presented in Figs. 9-10. Quantitative measures of the velocity magnitude acquired from a single position obtained central in the left ventricular outflow tract (LVOT) between the cups of the aortic valve are illustrated in Fig. 9. Fig. 10a shows the estimated velocity magnitudes through time for $10 \mathrm{~s}$ at the given position, where in total 8 full cardiac cycles were recorded. Fig. 10b shows the combined mean cardiac cycle velocities with their STDs. The mean peak systolic velocity (PSV) was $136 \mathrm{~cm} \mathrm{~s}^{-1}$ with an STD of \pm

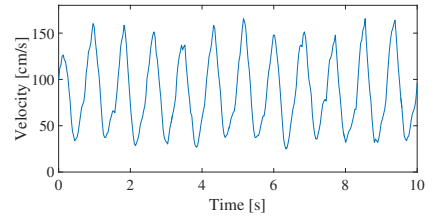

(a)

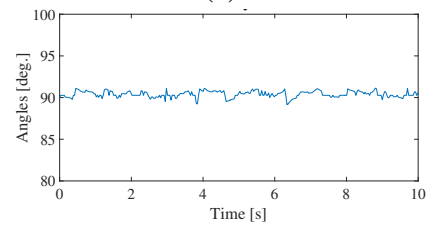

(c)

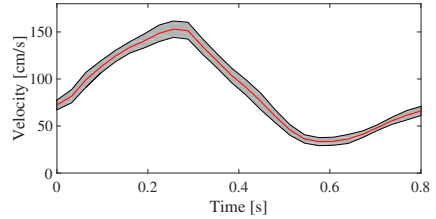

(b)

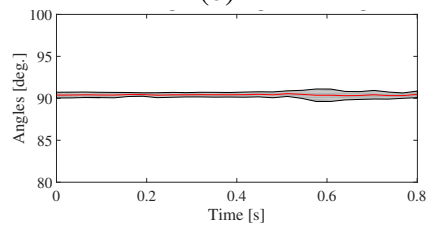

(d)
Figure 8: a) Estimated temporal velocity in the center of the vessel in a flow pump setup. b) Coherent alignment of the identified cycles, with mean cycle velocity (red curve) \pm one STD (grey area). c) Estimated temporal flow angle. d) Estimated mean flow angle (red curve) \pm one STD (grey area).

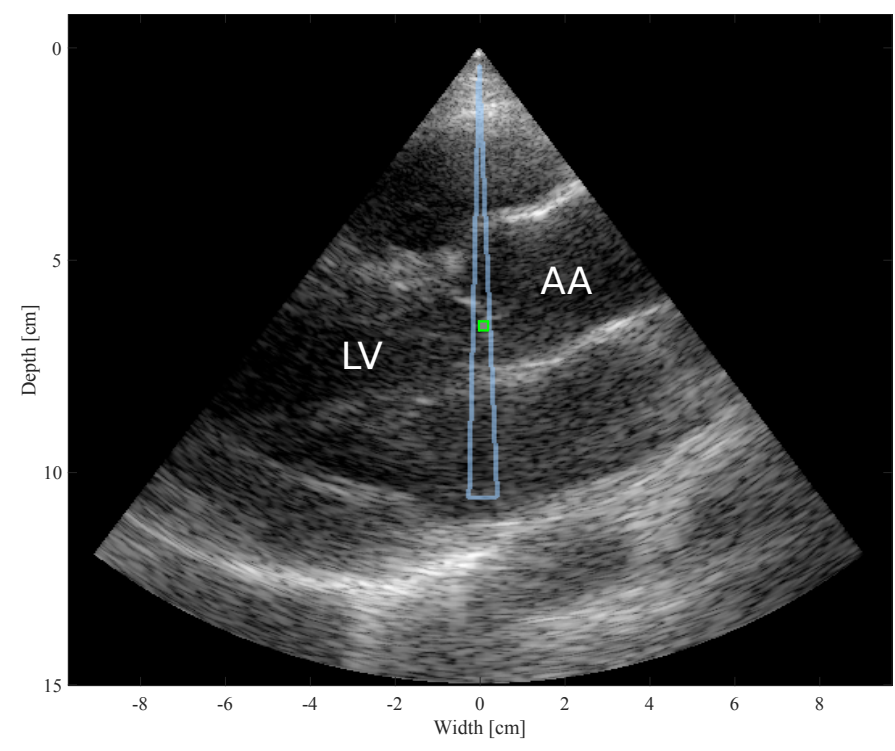

Figure 9: PLAX view of the ascending aorta. VFI was present in the outlined blue area and temporal velocity magnitudes were derived at the position denoted with the green square, just between the valves at $6.6 \mathrm{~cm}$ depth. $\mathrm{AA}=$ ascending aorta, $\mathrm{LV}=$ left ventricle.

16.9\%. In Fig. 11a, an obtained four-chamber view depicts the diastolic flow in the left ventricle of volunteer 2. A vortical flow is formed with the flow motion going along the free wall towards the apex and returning along the septum to the LVOT. In Fig 11b, the ascending aorta is shown in PLAX view with transverse laminar flow through the LVOT during systole.

\section{DISCUSSION}

The aim of this study was to implement phased array VFI on a commercial scanner for real-time rendering, which was successfully accomplished. Qualitative results of flow dynamics were shown in real-time on the scanner, whereas quantitative analyses were made off-line with an in-house built 


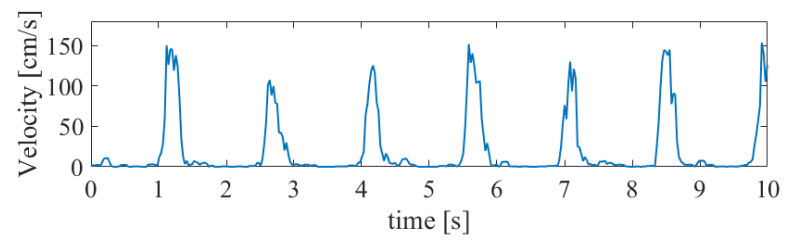

(a)

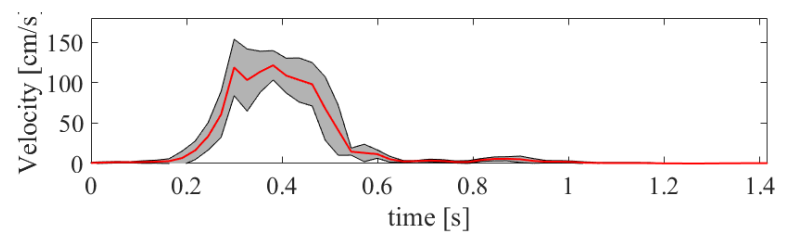

(b)

Figure 10: a): Estimated velocity magnitude in the ascending aorta for $10 \mathrm{~s}$. b): Combined mean cardiac cycle velocity magnitude (red curve) \pm one STD (grey area). 8 consecutive heart cycles were used in the statistics.

GUI. The presented results showed that the developed method can be used to estimate angle independent 2-D velocities of cardiac flow in the heart. The experimental validation showed that the bias and error in estimated flow angle are related to the relative beam-to-flow angle. The best estimates for the angle were found at fully transverse flow, where the beam-to-flow angle was $90^{\circ}$ and the influence from the axial component is lowest. Measurements in a pulsating known environment showed that PSV of $155 \mathrm{~cm} \mathrm{~s}^{-1}$ could be estimated with $9.0 \%$ precision. A bias in the experimental PSV estimation could not be determined, as the waveform was not expected to be fully developed at the measuring site. In vivo laminar transverse flow with PSV $>1 \mathrm{~m} \mathrm{~s}^{-1}$ was estimated with a precision of $\pm 16.9 \%$ in the ascending aorta, which is in agreement with previous reported velocity magnitudes [37]. Diastolic vortical flow was discovered in the left ventricle as previously demonstrated with other methods [21]. Flow in the ascending aorta has also been examined with linear arrays, where flow complexity and systolic backflow were estimated and showed similar flow patterns as the reported findings in this study [5], [38], [39]. The in-vivo measurements are also affected by the bias found in the estimator and depends on the beam-to-flow angles.

The flow-rig measurements presented in Fig. 5 showed that a symmetric bias profile was centered around a $90^{\circ}$ beamto-flow angle. Velocity biases similar to those presented in this work were also reported by Pihl et al. [28], and this should clearly be a topic for further optimization. Their study concluded that the change in bias as the beam-to-flow angle varied was related to the echo canceling. These findings might be related to the fact that the lateral wavelength is around an order of a magnitude larger than the axial wavelength. When a high PRF is chosen, such that a sufficient aliasing limit is present for the axial estimator, the estimated velocities in the lateral directions are only a fraction of the lateral aliasing limit. This means that the inter-frame movement of scatterer in the lateral direction is relatively small compared to the lateral wavelength, and hence, some of the signal from blood scatter movement might be removed during echo canceling. Future work should therefore focus on identifying if alternative echo canceling filters can be applied without causing the sharp rise in bias at a $90^{\circ}$ beam-to-flow angle, and whether it is related to the large difference in the lateral and axial wavelengths.

The removal of low frequency content in the lateral spectrum will bias the spectrum towards a higher mean lateral frequency affecting the velocity estimates towards higher values, since they are directly proportional to the mean lateral oscillation frequency. A method for improving performance is therefore to use directional lines, where the spectrum can be estimated and used in a self-calibrating scheme. The method yields more data for the velocity estimation at the expense of more beamforming calculations [40]. This has been employed in the new TO estimator to estimate the mean lateral oscillation frequency and thereby reduce the bias for the different measurement angles. The approach can also be combined with a full directional beamforming along the flow direction to improve on the bias [41], [42].

Quantification of flow in the ascending aorta in the PLAX view is usually not possible with conventional 1-D methods, as the flow direction in this view is close to $90^{\circ}$. A new insonation window, i.e. the PLAX view, could therefore advantageously be used to estimate the aortic flow, since the developed 2D VFI method provides angle independent estimates. Access to new sonification windows using 2-D angle independent VFI was likewise reported in a recent study of portal flow in the liver, where 1-D estimates were compared to 2-D VFI estimates obtained from various sonification windows [43].

The performance of the velocity estimates highly depends on the present signal-to-noise (SNR) ratio. For phased array deep tissue imaging, a high SNR can be challenging to obtain, as the acoustic attenuation scales with the travelled distance. One way to improve the SNR is by increasing the physical dimensions of the vibrating transducer elements, which would generate a higher acoustic output. However, phased array transducer are designed to fit in between the ribs, which hinders an expansion of the transducer dimension. Another approach to increase SNR is by increasing the acoustic output through the transmit voltage. Since the developed sequences are far from exceeding the intensity levels regarding MI and $I_{\text {spta }}$, an increased transmit voltage would result in a rise in the transducers' surface temperature. Due to the high PRF, which was required to provide a sufficient frame rate, the rise in surface temperature was the actual restricting factor for the two developed sequences. Increasing the SNR, and thereby, the performance of the velocity estimates, would require either a better heat dissipation on the transducer surface or lowering the PRF. Lowering the PRF is not without cost, as it would result in a lower frame rate and a reduced aliasing limit.

Another parameter, which has a high impact on the performance of the velocity estimator, is the echo cancelling. The purpose of the echo canceller is to filter out the signal from the surrounding tissue. In cases where non or little tissue movement is present, even simple low-pass filters perform adequate. However, when rapid tissue movement is present, 


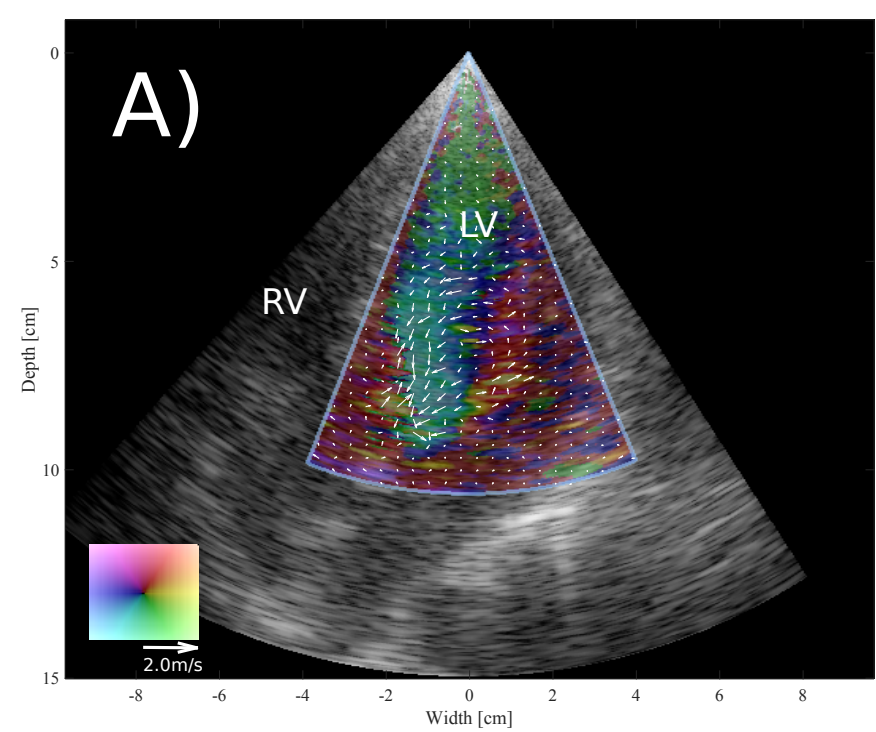

(a)

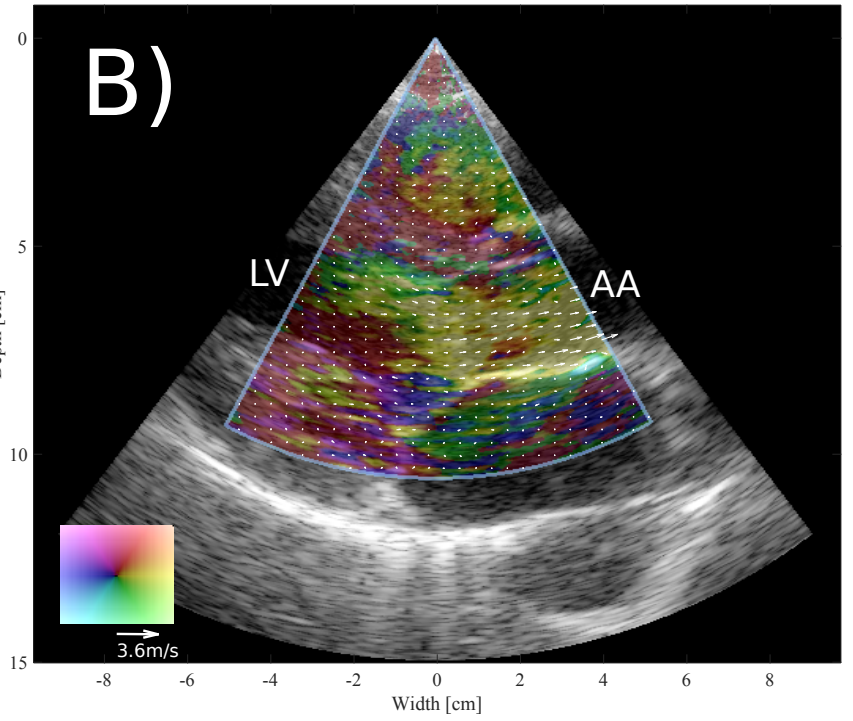

(b)

Figure 11: Examples of cardiac flow dynamics captured with the large FOV sequence. The direction and magnitude of the flow are given by the length of the arrow and the color of the superimposed VFI map. A) is obtained in a four-chamber view and depicts the vortical diastolic flow in the left ventricle. In B) the ascending aorta is shown in a PLAX view with transverse laminar flow through the LVOT during systole. $\mathrm{AA}=$ ascending aorta, $\mathrm{LV}=$ left ventricle, $\mathrm{RV}=$ right ventricle.

more advanced echo cancelling algorithms are required before a satisfying level of signal originating from tissue motion can be handed to the velocity estimator. In this study, tissue movement was only present in the experimental flow pump setup and in the in vivo measurements, although the complexity and magnitude of motion were expected to be highest in vivo. Based on the presented results from the pulsating setup, the applied echo canceller performed satisfying, as a low STD $<10 \%$ was seen during the largest tissue movement. Future work should therefore investigate the performance of different echo cancelling filters, as this could improve the precision of the estimates [44].

The developed VFI sequence had a penetration depth of $10.6 \mathrm{~cm}$ for flow and $15 \mathrm{~cm}$ for B-mode imaging. This can be sufficient for some scan views, as the method is angle independent compared to conventional 1-D spectral estimators. However, the FOV needs to be expanded in future work, especially when examinations on patients with a larger BMI are performed. An increasing estimation depth can be obtained at the cost of a sacrificing FOV and/or frame rate, since a prolonged travelling time is induced. The frame rate of 11 fps for the large FOV sequence was sufficient to capture the overall flow dynamics and directions in our studies, but would be insufficient for capturing short-lived flow dynamics. Further development should seek to balance this optimization task, or by using diverging/plane waves in transmit, such that several VFI estimation lines could be determined from one transmit direction.

Phased array VFI could be a powerful tool in echocardiography. The method can provide new parameters for cardiac flow, can visualize complex flow, and can offer new insonation windows for quantitative flow estimation. However, a larger study is warranted with a VFI phased array implementation providing real-time flow metrics on the scanner. First, the method should be validated in vivo on healthy volunteers. Second, cardiac VFI evaluations of patients with congenital heart defect and acquired cardiovascular disease should be conducted.

\section{CONCLUSION}

A real-time implementation of phased array VFI on a commercial ultrasound scanner (BK 5000, BK ultrasound, Herlev, Denmark) for cardiac applications was presented. Experimental validation of the developed method demonstrated that pulsating flow with peak velocities up to $1.5 \mathrm{~m} \mathrm{~s}^{-1}$ could be estimated with STD $<10 \%$. Furthermore, in vivo measurements showed that large FOV heart chamber flow dynamics could be visualized and that velocity magnitudes from the ascending aorta could be derived, even though the flow was fully transverse.

\section{ACKNOWLEDGMENT}

This work was supported by grant 82-2012-4 from the Danish National Advanced Technology Foundation and by BK Ultrasound Aps.

\section{REFERENCES}

[1] H. Baumgartner, J. Hung, J. Bermejo, J. B. Chambers, A. Evangelista, B. P. Griffin, B. Iung, C. M. Otto, P. A. Pellikka, and M. Quinones, "Echocardiographic assessment of valve stenosis: EAE/ASE recommendations for clinical practice," European Journal of Echocardiography, pp. 1-25, 2009.

[2] A. C. of Cardiology, "Acc/aha 2007 guidelines on perioperative cardiovascular evaluation and care for noncardiac surgery : executive summary : a report of the american college of cardiology/american heart association task force on practice guidelines (writing committee to revi," Anesth Analg., vol. 106, pp. 685-712, 2008. 
[3] A. S. of Anesthesiologists and S. of Cardiovascular Anesthesiologists Task Force on Transesophageal Echocardiography., "Practice guidelines for perioperative transesophageal echocardiography. an updated report by the american society of anesthesiologists and the society of cardiovascular anesthesiologists task force on transesophageal echocardiography." Anesthesiology, vol. 112, pp. 1084-1096, 2010.

[4] P. S. Douglas, M. J. Garcia, D. E. Haines, W. W. Lai, W. J. Manning, A. R. Patel, M. H. Picard, D. M. Polk, M. Ragosta, R. P. Ward, and R. B. Weiner, "Appropriate use criteria for echocardiography: A report of the american college of cardiology foundation appropriate use criteria task force, american society of echocardiography, american heart association, american society of nuclear cardiology, heart $\mathrm{f}$," Journal of the American College of Cardiology, vol. 57, no. 9, pp. 1126-1166, 2011.

[5] K. L. Hansen, H. Møller-Sørensen, J. Kjaergaard, J. T. Lund, M. M. Pedersen, T. Lange, J. A. Jensen, and M. B. Nielsen, "Analysis of systolic backflow and secondary helical blood flow in the ascending aorta using vector flow imaging," Ultrasound Med. Biol., vol. 42, no. 4, pp. 899-908, 2016.

[6] P. A. Picot and P. M. Embree, "Quantitative volume flow estimation using velocity profiles," IEEE Trans. Ultrason., Ferroelec., Freq. Contr., vol. 41, pp. 340-345, 1994

[7] J. Jensen, J. B. Olesen, M. B. Stuart, P. M. Hansen, M. B. Nielsen, and J. A. Jensen, "Vector velocity volume flow estimation: Sources of error and corrections applied for arteriovenous fistulas," Ultrasonics, vol. 70, pp. 136-146, 2016

[8] R. Steel, K. V. Ramnarine, F. Davidson, P. J. Fish, and P. R. Hoskins, "Angle-independent estimation of maximum velocity through stenoses using vector Doppler ultrasound," Ultrasound Med. Biol., vol. 29, no. 4, pp. 575-584, 2003.

[9] M. Markl, P. J. Kilner, and T. Ebbers, "Comprehensive 4D velocity mapping of the heart and great vessels by cardiovascular magnetic resonance," Journal of Cardiovascular MAgnetic Resonance, vol. 13, no. 1, p. 7, 2011.

[10] J. A. Jensen, "A new estimator for vector velocity estimation," IEEE Trans. Ultrason., Ferroelec., Freq. Contr., vol. 48, no. 4, pp. 886-894, 2001.

[11] M. Tanter, J. Bercoff, L. Sandrin, and M. Fink, "Ultrafast compound imaging for 2-D motion vector estimation: application to transient elastography," IEEE Trans. Ultrason., Ferroelec., Freq. Contr., vol. 49, pp. 1363-1374, 2002.

[12] J. A. Jensen and S. I. Nikolov, "Transverse flow imaging using synthetic aperture directional beamforming," in Proc. IEEE Ultrason. Symp., 2002, pp. $1488-1492$.

[13] B. Y. Yiu, S. S. Lai, and A. C. Yu, "Vector projectile imaging: timeresolved dynamic visualization of complex flow patterns." Ultrasound Med. Biol., vol. 40, no. 9, pp. 2295-2309, sept 2014.

[14] S. Ricci, L. Bassi, and P. Tortoli, "Real-time vector velocity assessment through multigate Doppler and plane waves," IEEE Trans. Ultrason. Ferroelec., Freq. Contr., vol. 61, no. 2, pp. 314-324, 2014.

[15] L. N. Bohs, B. J. Geiman, M. E. Anderson, S. C. Gebhart, and G. E. Trahey, "Speckle tracking for multi-dimensional flow estimation," Ultrasonics, vol. 38, pp. 369-375, 2000.

[16] H. Takahashi, H. Hasegawa, and H. Kanai, "Echo speckle imaging of blood particles with high-frame-rate echocardiography," Japanese Journal of Applied Physics, vol. 53, no. 07KF08, pp. 1-7, Jul 2014.

[17] S. Fadnes, M. S. Wigen, S. A. Nyrnes, and L. Lovstakken, "In vivo intracardiac vector flow imaging using phased array transducers for pediatric cardiology," Ieee Transactions on Ultrasonics Ferroelectrics and Frequency Control, vol. 64, no. 9, pp. 1318-1326, 2017.

[18] M. Wigen and L. Løvstakken, "In vivo three-dimensional intra-cardiac vector flow imaging using a 2D matrix array transducer," in Proc. IEEE Ultrason. Symp., 2016, pp. 1-4.

[19] C. A. Villagomez-Hoyos, M. B. Stuart, T. Bechsgaard, M. B. Nielsen, and J. A. Jensen, "High frame rate synthetic aperture vector flow imaging for transthoracic echocardiography," in Proc. SPIE Med. Imag., 2016.

[20] C. A. Villagomez-Hoyos, S. Holbek, M. B. Stuart, and J. A. Jensen, "High frame rate synthetic aperture 3D vector flow imaging," in Proc. IEEE Ultrason. Symp., 2016, pp. 1-4.

[21] F. Mehregan, F. Tournoux, S. Muth, P. Pibarot, R. Rieu, G. Cloutier, and D. Garcia, "Doppler vortography: a color doppler approach for quantification of the intraventricular blood flow vortices," Ultrasound Med. Biol., vol. 40, no. 1, pp. 210-221, January 2014.

[22] S. Ohtsuki and M. Tanaka, "The flow velocity distribution from the doppler information on a plane in three-dimensional flow," J. of Visualization, vol. 9, no. 1, pp. 69-82, 2006.

[23] D. Garcia, J. C. del Alamo, D. Tanne, R. Yotti, C. Cortina, E. Bertrand, J. C. Antoranz, E. Perez-David, R. Rieu, F. Fernandez-Aviles, and
J. Bermejo, "Two-dimensional intraventricular flow mapping by digital processing conventional color-doppler echocardiography images," IEEE Trans. Med. Imag., vol. 29, no. 10, pp. 1701-1713, 2010.

[24] J. A. Jensen, S. I. Nikolov, A. Yu, and D. Garcia, "Ultrasound vector flow imaging I: Sequential systems," IEEE Trans. Ultrason., Ferroelec., Freq. Contr., vol. 63, no. 11, pp. 1704-1721, 2016.

[25] — , "Ultrasound vector flow imaging II: Parallel systems," IEEE Trans. Ultrason., Ferroelec., Freq. Contr., vol. 63, no. 11, pp. 1722-1732, 2016.

[26] J. A. Jensen and P. Munk, "A new method for estimation of velocity vectors," IEEE Trans. Ultrason., Ferroelec., Freq. Contr., vol. 45, no. 3, pp. 837-851, 1998.

[27] J. Udesen and J. A. Jensen, "Investigation of Transverse Oscillation Method," IEEE Trans. Ultrason., Ferroelec., Freq. Contr., vol. 53, pp. 959-971, 2006.

[28] M. J. Pihl, J. Marcher, and J. A. Jensen, "Phased-array vector velocity estimation using transverse oscillations," IEEE Trans. Ultrason., Ferroelec., Freq. Contr., vol. 59, no. 12, pp. 2662-2675, 2012.

[29] S. Holbek, C. Ewertsen, H. Bouzari, M. J. Pihl, K. L. Hansen, M. B. Stuart, M. B. Nielsen, and J. A. Jensen, "Ultrasonic 3-D vector flow method for quantitative in vivo peak velocity and flow rate estimation," IEEE Trans. Ultrason., Ferroelec., Freq. Contr., vol. 64, no. 3, pp. 544 $554,2017$.

[30] S. Holbek, K. H. Lindskov, H. Bouzari, C. Ewertsen, M. B. Stuart, C. Thomsen, M. B. Nielsen, and J. A. Jensen, "Common carotid artery flow measured by 3-d ultrasonic vfi and validated with mri," Ultrasound Med. Biol., vol. 43, no. 10, pp. 2213-2220, 2017.

[31] H. Liebgott, J. Fromageau, J. E. Wilhjelm, D. Vray, and P. Delachartre, "Beamforming scheme for 2D displacement estimation in ultrasound imaging," EURASIP J. Adv. Signal Process., vol. 2005, no. 8, pp. 1212 $1220,2005$.

[32] M. Alessandrini, A. Basarab, L. Boussel, X. Guo, A. Serusclat, D. Friboulet, D. Kouamé, O. Bernard, and H. Liebgott, "A new technique for the estimation of cardiac motion in echocardiography based on transverse oscillations: A preliminary evaluation in silico and a feasibility demonstration in vivo," IEEE Trans. Med. Imag., vol. 33, no. 5, pp. 1148-1162, 2014

[33] A. C. H. Yu and Y. S. Yiu, "Apparatus for ultrasound flow vector imaging and methods thereof," 2014, patent Application PCT/CN2014/091035;

[34] C. Kasai, K. Namekawa, A. Koyano, and R. Omoto, "Real-Time TwoDimensional Blood Flow Imaging using an Autocorrelation Technique," IEEE Trans. Son. Ultrason., vol. 32, no. 3, pp. 458-463, 1985.

[35] J. A. Jensen, A. H. Brandt, and M. B. Nielsen, "Convex array vector velocity imaging using transverse oscillation and its optimization," IEEE Trans. Ultrason., Ferroelec., Freq. Contr., vol. 62, no. 12, pp. 2043 2053, 2015.

[36] FDA, "Information for manufacturers seeking marketing clearance of diagnostic ultrasound systems and transducers," Center for Devices and Radiological Health, United States Food and Drug Administration, Tech. Rep., 2008.

[37] J. E. Kvitting, T. Ebbers, L. Wigström, J. Engvall, C. L. Olin, and A. F. Bolger, "Flow patterns in the aortic root and the aorta studied with time-resolved, 3-dimensional, phase-contrast magnetic resonance imaging: implications for aortic valvesparing surgery," The Journal of Thoracic and Cardiovascular Surgery, vol. 127, no. 6, pp. 1602-1607, 2004.

[38] K. L. Hansen, H. Møller-Sørensen, J. Kjaergaard, M. B. Jensen, J. T. Lund, M. M. Pedersen, T. Lange, J. A. Jensen, and M. B. Nielsen, "Intraoperative vector flow imaging using ultrasound of the ascending aorta among 40 patients with normal, stenotic and replaced aortic valves," Ultrasound Med. Biol., vol. 42, no. 10, pp. 2414-2422, 2016.

[39] K. L. Hansen, H. Møller-Sørensen, J. Kjaergaard, M. B. Jensen, J. A. Jensen, and M. B. Nielsen, "Aortic valve stenosis increases helical flow and flow complexity: A study of intra-operative cardiac vector flow imaging," Ultrasound Med. Biol., vol. 43, no. 8, pp. 1607-1617, 2017.

[40] J. A. Jensen, "Directional transverse oscillation vector flow estimation," IEEE Trans. Ultrason., Ferroelec., Freq. Contr., vol. 64, no. 8, pp. 1194 1204, 2017.

[41] _ "Directional velocity estimation using focusing along the flow direction: I: Theory and simulation," IEEE Trans. Ultrason., Ferroelec., Freq. Contr., vol. 50, pp. 857-872, 2003.

[42] J. Jensen, C. A. Villagomez-Hoyos, M. B. Stuart, C. Ewertsen, M. B Nielsen, and J. A. Jensen, "Fast plane wave 2-D vector flow imaging using transverse oscillation and directional beamforming," IEEE Trans. Ultrason., Ferroelec., Freq. Contr., vol. 64, no. 7, pp. 1050-1062, 2017.

[43] A. H. Brandt, R. Moshavegh, K. L. Hansen, L. Lonn, J. A. Jensen, and M. Nielsen, "Vector flow imaging compared with pulse wave doppler for 
estimation of peak velocity in the portal vein," Ultrasound Med. Biol., vol. 44, no. 3, pp. 593-601, 2018.

[44] S. Bjærum, H. Torp, and K. Kristoffersen, "Clutter filter design for ultrasound color flow imaging," IEEE Trans. Ultrason., Ferroelec., Freq. Contr., vol. 49, pp. 204-209, 2002. 